\title{
Factors Associated with Heritable Pulmonary Arterial Hypertension Exert Convergent Actions on the miR-130/301-Vascular Matrix Feedback Loop
}

\author{
Thomas Bertero ${ }^{1, *}$, Adam L. Handen ${ }^{2}$ and Stephen Y. Chan ${ }^{2, *}$ (D) \\ 1 Université Côte d'Azur, CNRS UMR7284, INSERM U1081, IRCAN, Nice 06100, France \\ 2 Center for Pulmonary Vascular Biology and Medicine, Pittsburgh Heart, Lung, Blood, and Vascular \\ Medicine Institute, Division of Cardiology, Department of Medicine, University of Pittsburgh School of \\ Medicine and University of Pittsburgh Medical Center, Pittsburgh, PA 15213, USA; handena@pitt.edu \\ * Correspondence: bertero@unice.fr (T.B.); chansy@pitt.edu (S.Y.C.); Tel.: +33-493-377-753 (T.B.); \\ $+1-412-383-6990$ (S.Y.C.)
}

Received: 17 July 2018; Accepted: 1 August 2018; Published: 4 August 2018

\begin{abstract}
Pulmonary arterial hypertension (PAH) is characterized by occlusion of lung arterioles, leading to marked increases in pulmonary vascular resistance. Although heritable forms of PAH are known to be driven by genetic mutations that share some commonality of function, the extent to which these effectors converge to regulate shared processes in this disease is unknown. We have causally connected extracellular matrix (ECM) remodeling and mechanotransduction to the miR-130/301 family in a feedback loop that drives vascular activation and downstream PAH. However, the molecular interconnections between factors genetically associated with PAH and this mechano-driven feedback loop remain undefined. We performed systematic manipulation of matrix stiffness, the miR-130/301 family, and factors genetically associated with PAH in primary human pulmonary arterial cells and assessed downstream and reciprocal consequences on their expression. We found that a network of factors linked to heritable PAH converges upon the matrix stiffening-miR-130/301-PPAR $\gamma$-LRP8 axis in order to remodel the ECM. Furthermore, we leveraged a computational network biology approach to predict a number of additional molecular circuits functionally linking this axis to the ECM. These results demonstrate that multiple genes associated with heritable PAH converge to control the miR-130/301 circuit, triggering a self-amplifying feedback process central to pulmonary vascular stiffening and disease.
\end{abstract}

Keywords: heritable pulmonary hypertension; pulmonary arterial hypertension; genetic predisposition; microRNA; extracellular matrix; fibrosis

\section{Introduction}

Pulmonary hypertension $(\mathrm{PH})$ and a particularly severe subtype, pulmonary arterial hypertension $(\mathrm{PAH})$, are characterized by a complex panvasculopathy involving excessive proliferation and dysregulation of multiple cell types, as well as inflammation and fibrosis throughout the vasculature, leading to increased pulmonary arterial pressure and adverse remodeling of the pulmonary arterioles and often the right ventricle [1]. Exogenous injury (i.e., hypoxia, inflammation, infection) and various other illnesses are linked to $\mathrm{PH}$ development, and a growing list of mutations in specific pathogenic genes predispose to hereditary forms of $\mathrm{PH}$ [2]. Etiologies of $\mathrm{PH}$ are numerous, and five types of $\mathrm{PH}$ have been defined in an official World Health Organization (WHO) classification system [3], most recently revised in 2018. WHO Group 1 consists of individuals suffering from PAH, stemming either from idiopathic and hereditary forms or secondarily from co-morbidities such as congenital 
heart disease, autoimmune disease, drug and toxin exposure, and infections. WHO Groups $2-5$ can be triggered by a wide range of other conditions, such as left heart disease, chronic hypoxia, and thromboembolic disease, among others. PH and particularly PAH are highly morbid conditions.

Haploinsufficient loss-of-function mutations in the bone morphogenetic protein type 2 receptor (BMPR2) gene, a member of the transforming growth factor-beta (TGF- $\beta$ ) superfamily, account for over $80 \%$ of hereditary PAH cases and approximately $20 \%$ of idiopathic PAH cases [4]. Several mutations in other genes have been reported, accounting for at least a portion of the remainder of PAH heritability. Pathogenic mutations have been identified in genes encoding other components of the TGF- $\beta$ /bone morphogenetic protein (BMP) signaling pathways, including ACVRL1, ENG, GDF2 [5], $S M A D 9$, and $C A V 1$, providing compelling evidence for a central role of dysregulated BMP signaling in PAH pathogenesis [2]. More recently, mutations in genes not directly linked to TGF- $\beta$ /BMP signaling, including KCNK3 [6], TBX4 [7], as well as AQP1 [5], ATP13A3 [5], GCN2 [8], and SOX17 [5] have been linked to PAH; many of these mutations were identified by whole genome sequencing. Moreover, a locus in the CBLN2 gene was linked to PAH using a genome-wide association study [9]. Yet, beyond their immediate relation to TGF- $\beta$ / BMP signaling for some, contribution of these genetic factors to $\mathrm{PAH}$ progression, particularly in relation to one another, remains incompletely described [2].

Recently, we [10] and others [11] observed that vascular stiffness and extracellular matrix (ECM) remodeling promotes pulmonary vascular dysfunction early in disease pathogenesis. In general, ECM remodeling is a complex process, governed through a balance between ECM protein deposition and degradation, as well as ECM assembly via collagen crosslinking enzymes such as lysyl oxidase (LOX). Decreased vessel wall compliance (i.e., stiffness) occurs in various forms of $\mathrm{PH}$ and is an important contributor and index of disease progression [12-16]. Vascular stiffness is determined by vascular tone and the quantity and composition of the ECM. While regulators of vascular tone have been very well studied, less is known about effectors and mechanisms that might regulate vascular stiffness by modulating ECM production/composition. Although anatomic location may dictate distinct effects on disease progression, stiffness in both the proximal and distal pulmonary arterial tree is important for overall pathogenesis [11] but the molecular mechanisms controlling these processes are only beginning to be appreciated.

The structural alterations in the pulmonary vasculature are controlled by activated vascular cells, which in PAH exhibit metabolic reprogramming and consequent hyperproliferative, migratory, and invasive capabilities. Given their inherent pleiotropic actions to repress multiple gene targets simultaneously, microRNAs (miRNAs) are essential mediators of multiple cellular processes and may be ideal candidates to provide comprehensive and integrated control of PAH. Previously, we utilized a network-based approach to investigate the role of miRNAs in the integrated control of PH pathogenesis and described the proliferative and vasoconstrictive actions of miR-130/301 family in $\mathrm{PH}[17,18]$. Beyond these functions, we also reported a prominent component of their related gene targets associated with ECM biology. We established vascular matrix stiffness as an early, pervasive driver of many types of $\mathrm{PH}$ [10], controlled by mechanoactivation of two related transcriptional co-activators, YAP (Yes Associated Protein 1) and TAZ (Transcriptional Coactivator with PDZ-Binding Motif), factors that are known to regulate proliferation, survival, organ size, and the ECM. We found that downstream induction of the microRNA-130/301 family further engages a network of related gene targets including the PPAR $\gamma$-APOE-LRP8 axis that regulates LOX activity to coordinate ECM remodeling and sustain matrix stiffening in $\mathrm{PH}$. Yet, whether the actions of these seemingly disparate factors that are genetically associated with PAH converge to activate this YAP/TAZ-miR-130/301 feedback loop remains undefined. Consequently, in this study we investigated whether factors that are genetically associated with PAH can influence the miR-130/301-ECM remodeling feedback loop, as well as whether the miR-130/301-ECM remodeling feedback loop controls expression of those same factors. 


\section{Results}

\subsection{Matrix Stiffening Modulates the Expression of Several Factors Genetically Associated with PAH}

Since factors with specific genetic mutations drive hereditary $\mathrm{PAH}$, and since matrix stiffening is an early driver of this disease, we hypothesized that ECM stiffening also controls the expression of these factors. To determine whether mechanical or physical cues conveyed by ECM stiffness modulate the expression of these factors, we cultivated pulmonary arterial adventitial fibroblasts (PAAFs), endothelial cells (PAECs), and smooth muscle cells (PASMCs) on ECM representing physiologic (soft; $1 \mathrm{kPa}$ ) or pathophysiologic (stiff; $12 \mathrm{kPa}$ ) levels of vascular stiffness [19]. By RT-qPCR, transcript expression levels of genes associated with PAH were quantified (Figure 1). Matrix stiffening significantly increased CBLN2 and decreased ACVRL1, BMPR2, GDF2, and KCNK3 across all vascular cell types tested: PAAFs (Figure 1A), PAECs (Figure 1B), and PASMCs (Figure 1C). Conversely, cell type context-specificity was noted in the alterations of other genes. This was the case for GCN2, a gene related to the amino acid stress response pathway, which was downregulated in PAAFs, but upregulated in PAECs, by stiff matrix. Alternatively, in PAECs and PASMCs, stiff matrix upregulated CAV1 but downregulated this gene in PAAFs (Figure 1D).

A
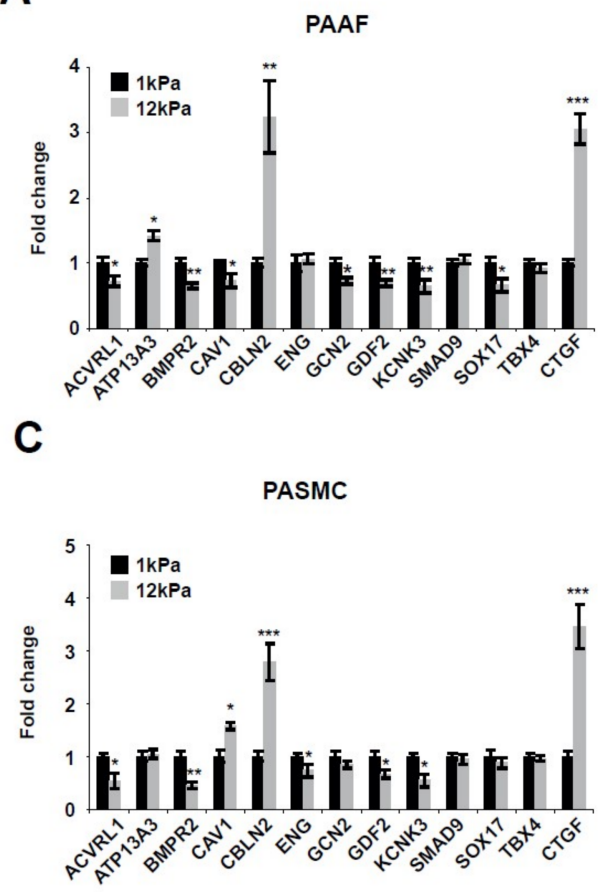

B
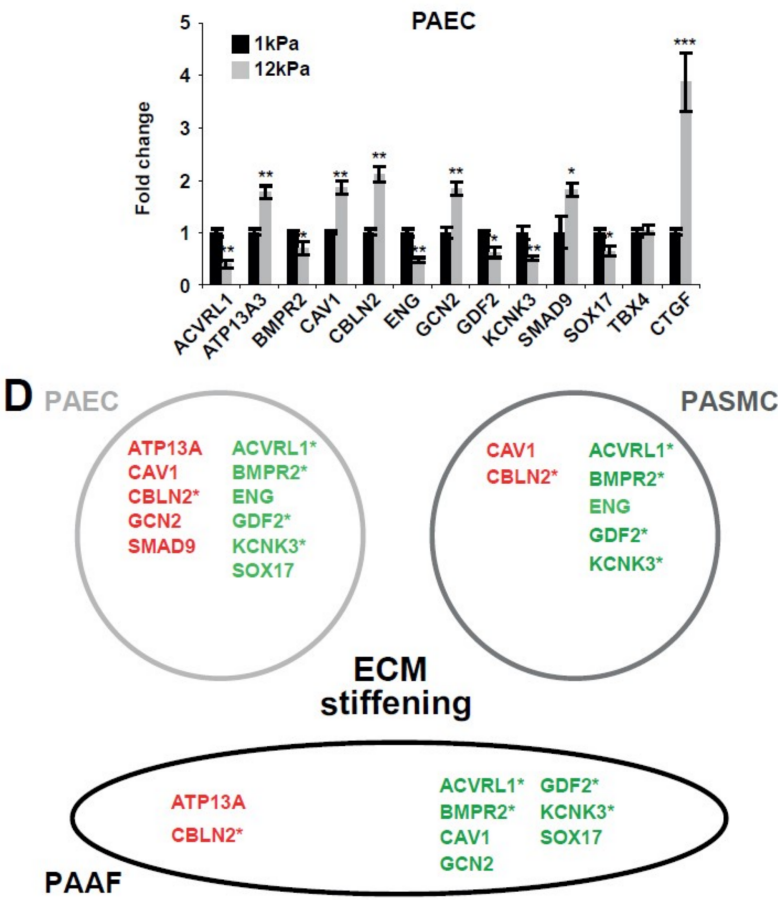

Figure 1. Factors genetically associated with PAH are responsive to matrix stiffening. (A-C) Pulmonary arterial cells were plated on soft $(1 \mathrm{kPa})$ or stiff $(12 \mathrm{kPa})$ hydrogel for $48 \mathrm{~h}$. Expression level of factors genetically associated with PAH in PAAFs (A), PAECs (B), and PASMCs (C) were analyzed by RT-qPCR. Mean expression in control groups $(1 \mathrm{kPa})$ was assigned a fold change of 1 , to which relevant samples were compared. Data are expressed as mean $\pm \mathrm{SD}\left({ }^{*} p<0.05,{ }^{* *} p<0.01,{ }^{* * *} p<0.001\right)$ of at least three independent experiments. Paired samples were compared using a 2-tailed Student's $t$-test. (D) Schematic of the main results. Red: upregulated genes; Green: downregulated genes; * denote consistent and significant modulation in the three vascular cell types.

In sum, the majority (10 out of 12) of tested factors were found to be sensitive to mechanical cues conveyed by the ECM, suggesting that, in addition to genetic mutation, the levels of these PAH drivers are dynamically regulated by biophysical triggers. 


\subsection{The miR-130/301 Family is Modulated by Several Factors Genetically Associated with PAH}

Because matrix stiffness modulates genes linked to hereditary PAH (Figure 1) as well as miR-130/301 family members [10], we wanted to determine whether these factors genetically associated with PAH control expression of the miR-130/301 family (Figure 2). First, by RT-qPCR, we confirmed the robust knockdown of these factors after specific siRNA sequence transfection in human PAAFs, PAECs, and PASMCs (Figure S1). Adding to findings from our original published report [18], such knockdown of BMPR2, CAV1, GDF2, and SOX17 in turn increased miR-130/301 family member expression, while inhibition of CBLN2 decreased miR-130/301 family members in human PAAFs (Figure 2A), PAECs (Figure 2B), and PASMCs (Figure 2C). In addition, we found that KCNK3 knockdown increased miR-130/301 family expression in both PAAFs and PAECs, while knockdown of ENG and ACVRL1 increased miR-130/301 members only in PAAFs. Moreover, ATP13A3 knockdown increased miR-130/301 members in both PAECs and PASMCs.

Together, these results indicate the broad convergence of genetically associated PAH factors on coordinated regulation of this key microRNA family (Figure 2D).

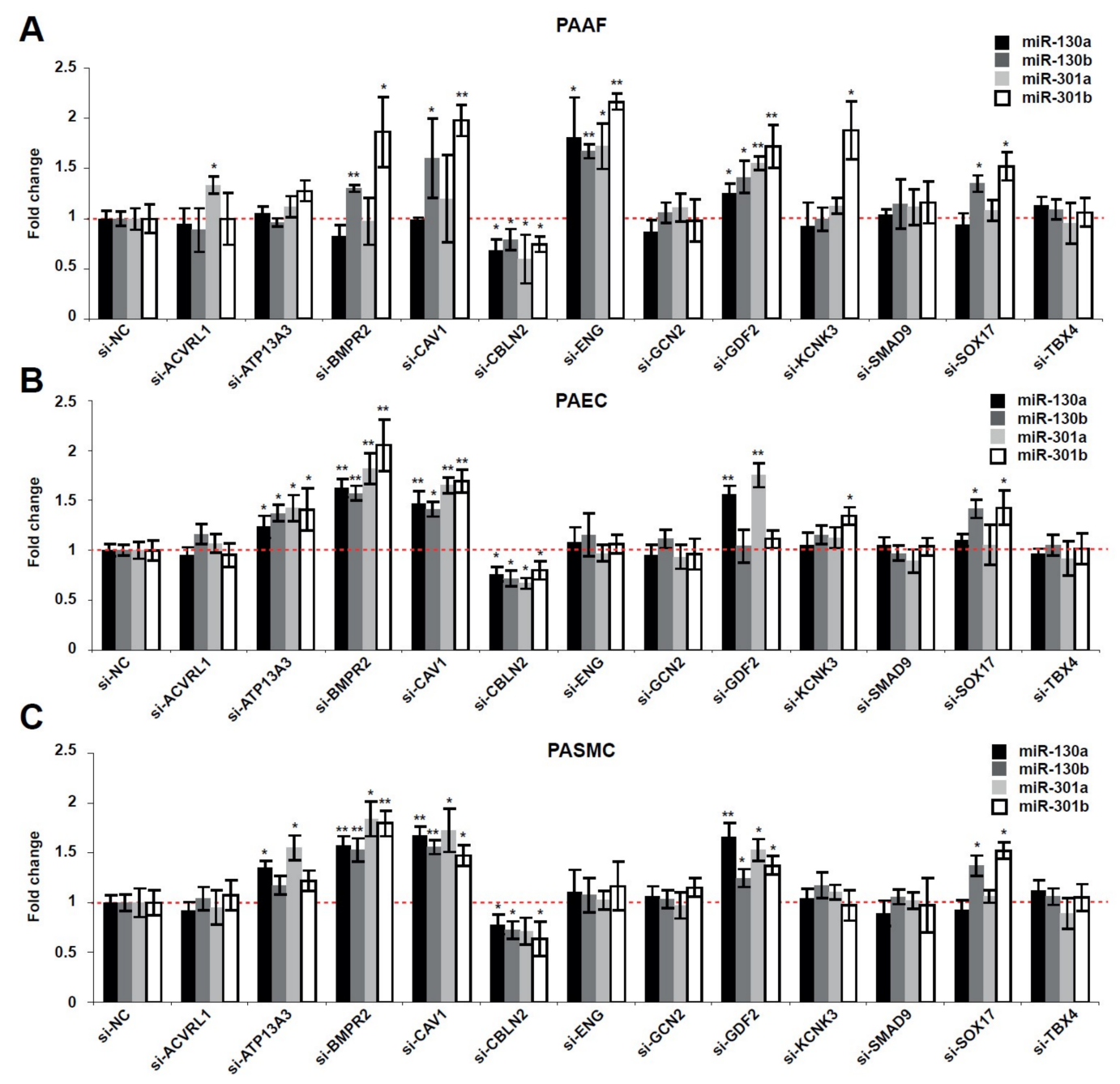

Figure 2. Cont. 


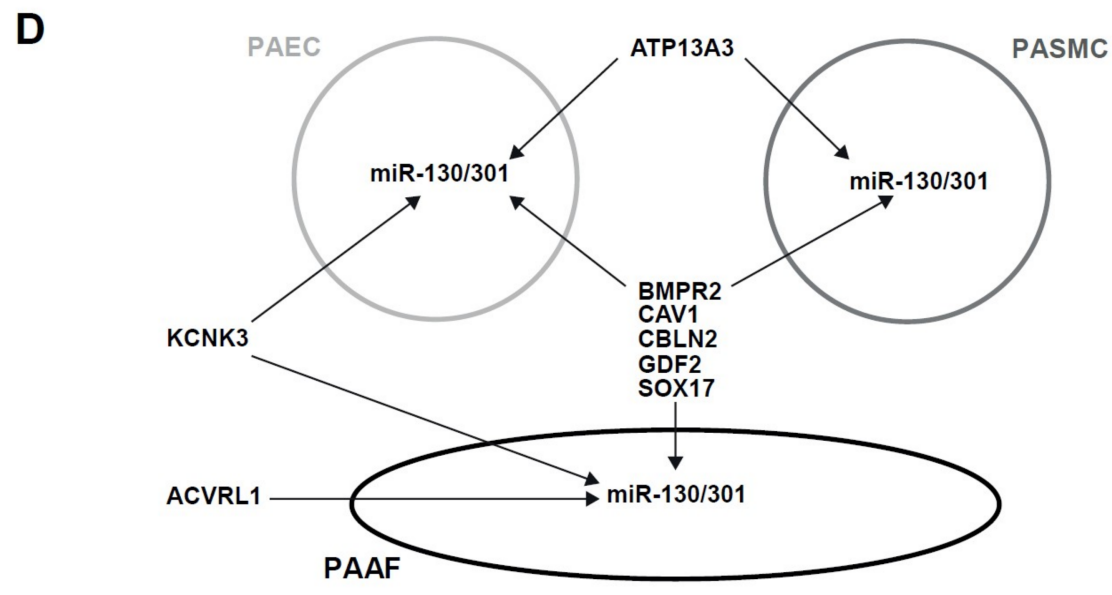

Figure 2. Factors genetically associated with PAH modulate the expression of miR-130/301 family members. (A-C) Following transfection with the indicated siRNA, transcript levels of miR-130/301 members were analyzed by RT-qPCR in PAAFs (A), PAECs (B), and PASMCs (C). Mean expression of a given miRNA in the control group (si-NC) was assigned a fold change of 1 , to which corresponding miRNA levels in experimental groups were compared. Data are expressed as mean $\pm \mathrm{SD}\left({ }^{*} p<0.05\right.$, $* *<0.01)$ of three independent experiments. One-way ANOVA and post-hoc Tukey tests were used for group comparisons. (D) Schematic of the main results.

\subsection{The miR-130/301 Family Controls the Vascular Expression of Factors Associated with Hereditary PAH}

MicroRNA-mediated feed-back and feed-forward loops are recurrent network motifs to enhance the robustness of gene regulation in mammalian genomes [20]. Thus, given the regulation of miR-130/301 by factors associated with hereditary PAH, we investigated whether the miR-130/301 family reciprocally controls the expression of these factors in PA vascular cells (Figure 3). First, we performed an in silico analysis of miR-130/301 target genes, using the well-validated microRNA target prediction algorithm Targetscan [21] and revealing canonical and conserved binding sites for miR-130/301 in the 3' untranslated regions (3'UTR) of ATP13A3, BMPR2, and SMAD9 transcripts. To mechanistically confirm these predictions, we performed gain-of-fuction experiments in vitro by forcing expression of miR-130a via transfection of oligonucleotide mimics in vascular cells cultivated in soft matrix (1 kPa). Across PAAFs (Figure 3A), PAECs (Figure 3B), and PASMCs (Figure 3C), such mimics decreased the transcript levels of $A T P 13 A 3$ and BMPR2 as well as ACVRL1 and ENG. Mimics also increased CBLN2 transcript. Not all effects of miR-130a were consistent across cell types, as forced miR-130a expression decreased CAV1 and SMAD9 transcript levels only in PAAFs and PASMCs, decreased GDF2 transcript only in PAECs and PASMCs, and increased expression of KCNK3 solely in PASMCs. Loss-of-fuction experiments were also performed in cells cultivated in stiff matrix (12 $\mathrm{kPa}$ ), whereby inhibition of the entire miR-130/301 family was achieved using a specifically designed short locked nucleic acid oligonucleotide ("tiny LNA") with antisense complementarity only to the seed sequence of this microRNA family [18]. Converse to the effects of forcing miR-130a expression, inhibition of the miR-130/301 family in stiff matrix increased the transcript levels of ACVRL1, ATP13A, $B M P R 2$, and ENG in PAAFs (Figure 3D), PAECs (Figure 3E), and PASMCs (Figure 3F), but decreased the expression of CBLN2. 


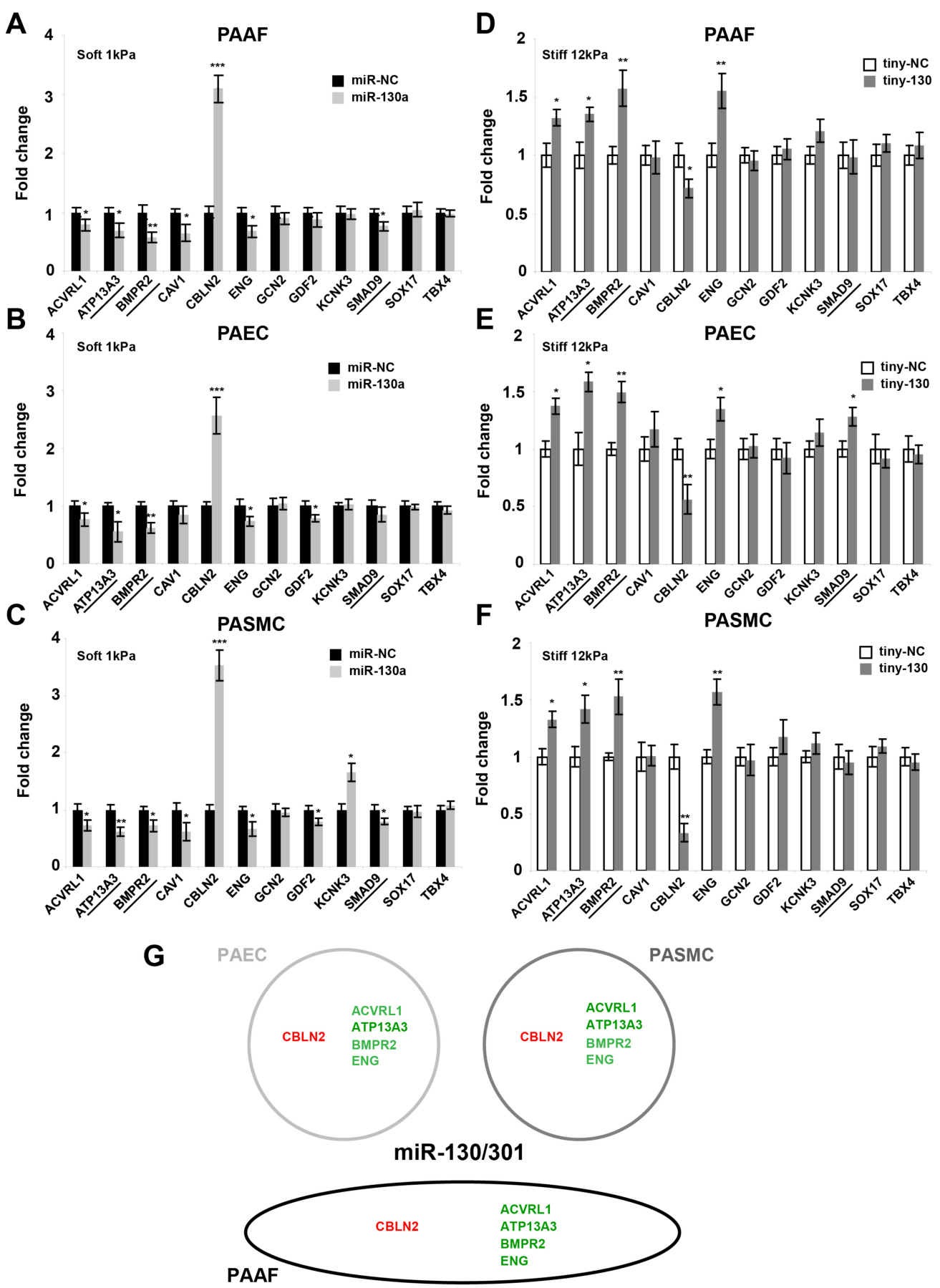

Figure 3. The miR-130/301 family modulates the expression of factors genetically associated with PAH. Following transfection with the indicated miR-130a mimic (A-C), miR-130/301 inhibitor (tiny-130) (D-F), or related scrambled controls, transcript levels of factors genetically associated with PAH were analyzed by RT-qPCR in PAAFs, PAECs, and PASMCs. Underlined genes: Predicted targets of miR-130/301 members (TargetScan). Mean expression in control groups (si-NC) was assigned a fold change of 1 , to which relevant samples were compared. Data are expressed as mean $\pm \operatorname{SD}\left({ }^{*} p<0.05,{ }^{* *}\right.$ $\left.p<0.01,{ }^{* * *} p<0.001\right)$ of three independent experiments. Paired samples were compared using a 2-tailed Student's t-test. (G) Schematic of the main results. Red: upregulated genes. Green: downregulated genes, upon miR-130a forced expression. 
Taken together, these results demonstrate the pervasive influence of miR-130/301 family in controlling factors genetically linked to PAH, indicating that the miR-130/301 family often is both necessary and sufficient to modulate some of these stiffness-dependent factors (Figure 3G).

\subsection{Factors Genetically Associated with PAH Broadly Impact the PPAR $\gamma-A p o E-L R P 8-M a t r i x$ Remodeling} Axis

Recently, we described the miR-130/301-PPAR $\gamma$-APOE-LRP8-matrix remodeling loop as a central positive feedback mechanism in PAH and fibrotic diseases $[10,22]$. Given our current findings that indicate mutual and reciprocal regulation between miR-130/301 members and factors associated with hereditary PAH (Figures 2 and 3), we investigated whether factors genetically linked to PAH interact with this central pathogenic axis (Figure 4). We found that siRNA-mediated knockdown of KCNK3 increased LOX transcript expression in PAAFs (Figure 4A), PAECs (Figure 4B), and PASMCs (Figure 4C). Moreover, inhibition of ACVRL1, ATP13A3, BMPR2, CAV1, CBLN2, ENG, GCN2, GDF2, TBX4, and SOX17 all differentially modulated the expression of the factors contributing to the central PPAR $\gamma$-APOE-LRP8 axis. While siRNA-mediated knockdown of GCN2 and TBX4 modulated transcript expression of specific fibrillar collagen genes (COL1A1 and COL3A1) and LOX, inhibition of others only modified a subset of these downstream matrix remodeling genes. Namely, inhibition of $A C V R L 1, B M P R 2$, and GDF2 increased transcript expression of fibrillar collagen genes (COL1A1 and COL3A1), while inhibition of CAV1 and CBLN2 decreased LOX.

These findings reveal that the network of factors linked to hereditary PAH all regulate the miR-130/301-PPAR $\gamma$-LRP8 axis in various vascular cell types, thus controlling downstream collagen and collagen remodeling genes crucial to ECM stiffening (Figure 4D). As such, these findings define the unique central importance of this ECM-relevant molecular circuitry among seemingly disparate genetic factors in $\mathrm{PAH}$, thus solidifying the principle of vascular stiffness as a primary driver of PAH. 

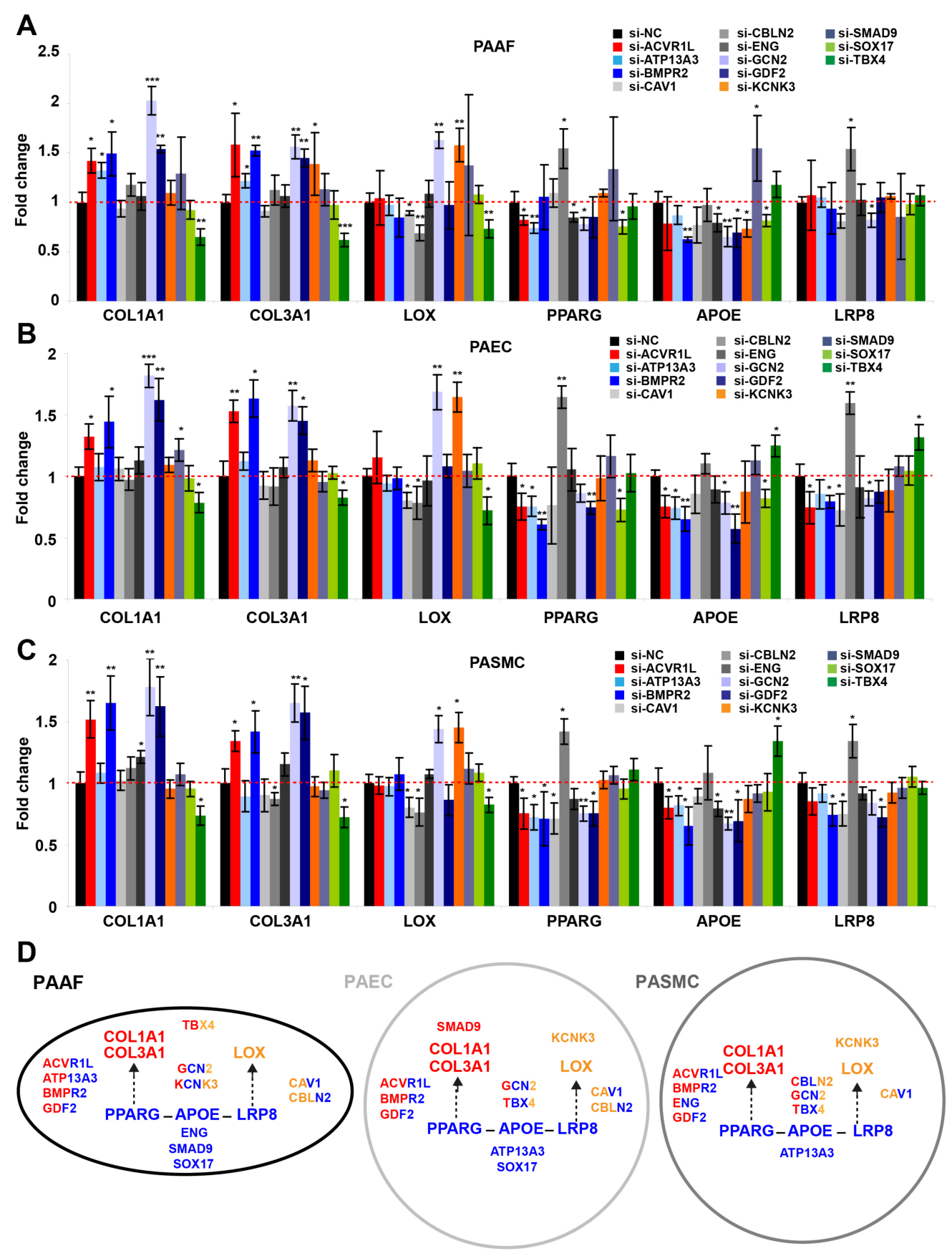

Figure 4. Upstream factors linked to hereditary PAH modulate the PPAR $\gamma$-APOE-LRP8 axis, which in turn controls pulmonary vascular matrix stiffening. (A-C) Following transfection with the indicated siRNAs, transcripts related to the PPAR $\gamma$-APOE-LRP8-matrix remodeling axis were analyzed by RT-qPCR in PAAFs (A), PAECs (B), and PASMCs (C). Mean expression of a given miRNA in the control group (si-NC) was assigned a fold change of 1, to which corresponding miRNA levels in experimental groups were compared. Data are expressed as mean $\pm \operatorname{SD}\left({ }^{*} p<0.05,{ }^{* *} p<0.01\right.$, *** $p<0.001$ ) of three independent experiments. One-way ANOVA and post-hoc Tukey tests were used for group comparisons. (D) Schematic of the main results. In each cell type, the central downstream PPAR $\gamma$-APOE-LRP8-LOX-collagen axis is drawn. Factors genetically linked to PAH are strategically placed next to the portion of the axis which they regulate; font is also color-coded based on these connections. Blue: genes related to the PPAR $\gamma$-APOE-LRP8 axis; Red: genes related to fibrillar collagen; Orange: genes related to LOX. 
2.5. A Computational Network Biology Approach Predicts Additional Molecular Pathways Linking Genes of Heritable PAH to the miR-130/301-ECM Axis

Based on the increasingly apparent convergent actions of factors important in hereditary PAH on miR-130/301 pathobiology and vascular stiffening, we endeavored to utilize a computational network biology approach to predict and visualize more comprehensively the functional interactions among these factors. To do so, we first constructed a PH gene network and fibrosis network, adapted from our prior published methodology [18]. In this case, for the PH network, we curated human genes known to play a role in PH from a literature search [18] and from the Pulmonary Arterial Hypertension Knowledge Base (PAHKB) [23]. We mapped functional interactions among these genes and with first-degree interactors via incorporating gene and protein interaction data from public databases, including DIP, BioGRID, Corum, InnateDB, IntAct, MINT, and MatrixDB [24-30]. A Fibrosis Network was similarly adapted from our prior report [22], with updated modifications including YAP/TAZ-dependent fibrotic factors in PAH [10]. Functional interactions of the known factors genetically associated with PAH and known target genes of miR-130/301 were then mapped into the PH and Fibrosis Networks (Figure 5A, Table S1). Importantly, and consistent with our experimental data (Figures 1-4), a key overlap was observed among the PH and Fibrosis Networks, encompassing known miR-130/301 targets (LRP6, LRP8, PPAR $\gamma$, and SP1), specific PAH hereditary factors (ENG, CAV1, SMAP9, and EIF2AK4), as well as multiple genes functionally related to miR-130/301 in PAH (COL1A1, COL3A1, LOX, YAP1, TAZ, and FN1). Importantly, while a number of these interactions were validated independently by our experimental studies (Figures 1-4), this intersection also provided insight into numerous novel connections, thus offering a more complete functional roadmap for deciphering the relationships among these factors. Specifically, there were a number of genes implicated in these relationships that did not factor into either the pre-established $\mathrm{PH}$ or Fibrosis Networks (outer ring of genes, Figure 5A). While gene set enrichment analysis (GSEA) [31] of the PH and Fibrosis Networks predictably identified pathways involved in fibrosis and vascular stiffness (i.e., TGF- $\beta$ /BMP signaling among others), GSEA of the non-PH/non-fibrosis genes indicated a number of novel pathways, such as immune activation, cell cycle checkpoint regulation, and DNA damage [32,33], never associated with miR-130/301, vascular stiffening, or several of these factors of hereditary PAH (Figure 5B, Tables S2 and S3). Thus, beyond the experimentally confirmed relationships defined in Figures 1-4, computational network predictions implicate a range of novel functional connections and pathways mediating the association of the miR-130/301-ECM axis with the molecular origins of hereditary PAH. 


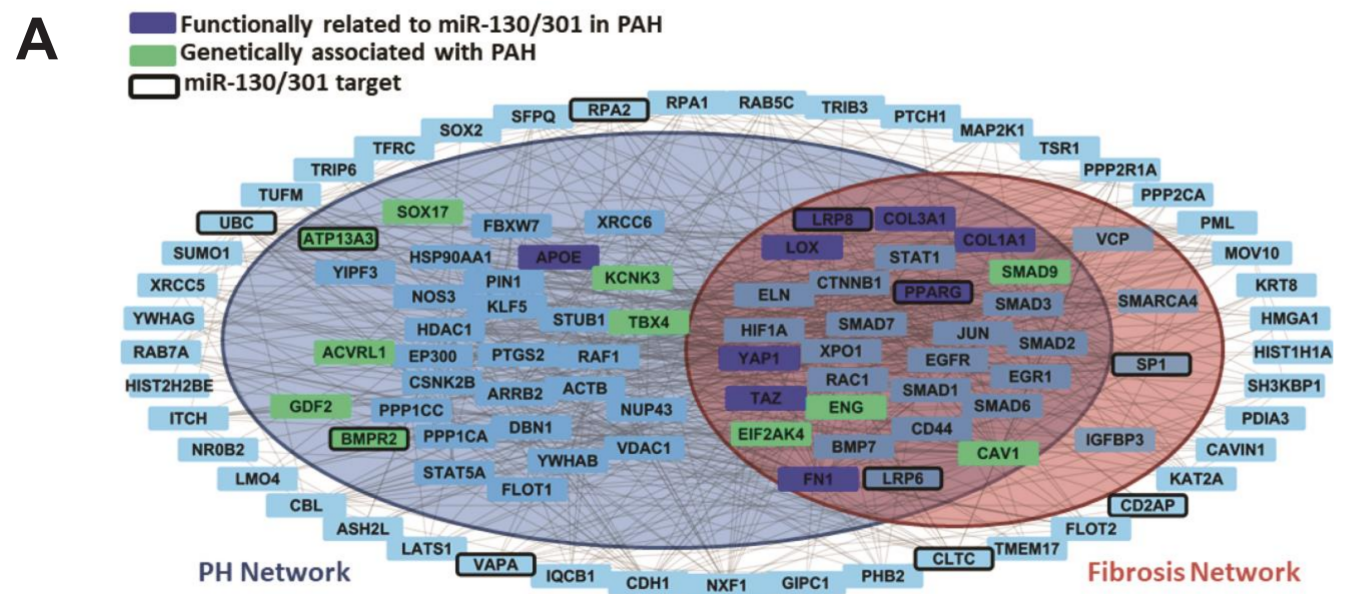

\section{B Expanded Network Enrichments}

Outer Enrichments

\begin{tabular}{|c|c|c|c|c|c|c|c|}
\hline Database & Pathway & $\begin{array}{l}\text { FDR } P- \\
\text { value }\end{array}$ & Size & Database & Pathway & $\begin{array}{l}\text { FDR } P \text { - } \\
\text { value }\end{array}$ & Size \\
\hline REACTOME & Signal Transduction & $4.73 \times 10^{-26}$ & 2598 & GO & protein binding & $6.75 \times 10^{-7}$ & 9409 \\
\hline GO & protein binding & $2.95 \times 10^{-20}$ & 9409 & REACTOME & $\begin{array}{l}\text { Gene expression } \\
\text { (Transcription) }\end{array}$ & $4.54 \times 10^{-5}$ & 1330 \\
\hline REACTOME & $\begin{array}{l}\text { Signaling by TGF-beta } \\
\text { family members } \\
\text { Gene expression }\end{array}$ & $5.35 \times 10^{-18}$ & 101 & REACTOME & $\begin{array}{l}\text { Listeria } \\
\text { monocytogenes entry } \\
\text { into host cells }\end{array}$ & $4.54 \times 10^{-5}$ & 19 \\
\hline REACTOME & (Transcription) & $3.92 \times 10^{-17}$ & 1330 & REACTOME & Immune System & $4.57 \times 10^{-5}$ & 1945 \\
\hline REACTOME & $\begin{array}{l}\text { Generic Transcription } \\
\text { Pathway }\end{array}$ & $1.15 \times 10^{-16}$ & 1074 & REACTOME & $\begin{array}{l}\text { Spry regulation of } \\
\text { FGF signaling }\end{array}$ & $4.76 \times 10^{-5}$ & 14 \\
\hline REACTOME & Immune System & $1.42 \times 10^{-15}$ & 1945 & REACTOME & $\begin{array}{l}\text { Cell Cycle } \\
\text { Checkpoints }\end{array}$ & $5.02 \times 10^{-5}$ & 270 \\
\hline REACTOME & $\begin{array}{l}\text { RNA Polymerase II } \\
\text { Transcription }\end{array}$ & $2.48 \times 10^{-15}$ & 1196 & REACTOME & Signal Transduction & $5.11 \times 10^{-5}$ & 2598 \\
\hline REACTOME & $\begin{array}{l}\text { Diseases of signal } \\
\text { transduction }\end{array}$ & $9.32 \times 10^{-15}$ & 359 & REACTOME & $\begin{array}{l}\text { RNA Polymerase II } \\
\text { Transcription }\end{array}$ & $5.11 \times 10^{-5}$ & 1196 \\
\hline KEGG & PATHWAYS_IN_CANCER & $1.09 \times 10^{-14}$ & 325 & REACTOME & $\begin{array}{l}\text { Generic Transcription } \\
\text { Pathway }\end{array}$ & $5.3 \times 10^{-5}$ & 1074 \\
\hline GO & $\begin{array}{l}\text { positive regulation of } \\
\text { transcription by RNA } \\
\text { polymerase II }\end{array}$ & $3.09 \times 10^{-14}$ & 1095 & REACTOME & $\begin{array}{l}\text { Negative regulation } \\
\text { of FGFR3 signaling }\end{array}$ & $5.44 \times 10^{-5}$ & 23 \\
\hline
\end{tabular}

Figure 5. A network bioinformatics approach predicts the complex and overlapping relationships among factors genetically associated with PAH with known miR-130/301 target genes, PH genes, and fibrosis genes. (A) Network model. Gray lines indicate an interaction between two genes. Genes in dark blue are functionally related to miR-130/301 members in PAH, and genes in green are associated with heritable PAH. Genes with a thickened border are predicted targets of miR-130/301 in TargetScan [21]. Genes found in the larger blue circle are common to the PH Network, and those found in the orange circle are common to the Fibrosis Network. (B) In the left table, top-ranked pathways via gene set enrichment analysis (GSEA) are listed of genes found in the PH and Fibrosis Networks interacting with known miR-130/301 target genes and factors genetically associated with PAH (e.g., genes located in the inner colored circles of $(\mathbf{A})$ ). In the right table, top-ranked pathways are listed of genes interacting with miR-130/301 target genes and factors associated with heritable PAH found outside the PH/Fibrosis Networks (e.g., genes located outside the colored circles of (A)).

\section{Discussion}

ECM stiffening, remodeling, and downstream mechanotransduction are recognized as causative drivers of multiple diseases, including PAH, by sustaining cell activities including proliferation, migration, apoptosis resistance, and metabolic rewiring [34]. Particularly in PAH, matrix remodeling and downstream vascular stiffening are increasingly appreciated as causative drivers of the disease process, promoted at least in part by the mechanosensitive miR-130/301 family and perivascular matrix remodeling and stiffening [10]. In this study, we found that seemingly disparate genetic drivers of PAH may activate this self-sustaining, positive feedback loop. These results are significant, as they emphasize the causative importance of this pathway in PAH pathogenesis and reinforce the 
attractive potential of developing tailored therapies for this molecular circuit of fibrosis. Moreover, our results indicate that, along with the TGF/BMP pathway, the miR-130/301-ECM axis serves as a central pathway where the functions of multiple factors of heritable PAH converge.

In light of the as-of-yet unexplained observation of incomplete genetic penetrance in heritable $\mathrm{PAH}$, it has been proposed that the development of $\mathrm{PH}$ may originate from the interaction of a genetic state predisposed to disease along with one or more inciting stimuli (a "multiple-hit hypothesis") [35]. More recently, PAH patients have been reported to carry multiple gene mutations in at least two distinct pathogenic loci [36,37], furthering the hypothesis that these multiple hits may originate from the complex genetic inheritance of several mutations. Our findings of convergent, miR-130/301-specific actions of seemingly disparate factors relevant to hereditary PAH now offer new insights into this multiple-hit hypothesis. Specifically, our findings could suggest that higher penetrance or more severe manifestation of disease may be observed in heritable $\mathrm{PAH}$, particularly when multiple gene mutations converge upon the central miR-130/301-ECM axis. Alternatively, future work will be imperative to determine if other genomic polymorphisms associated with ECM biology are also linked to heritable $\mathrm{PAH}$ and could contribute to disease manifestation. In addition, considering that the miR-130/301 members are upregulated by other acquired triggers of $\mathrm{PH}$ such as stiffness, hypoxia, and inflammatory cytokines $[10,18]$, our work suggests that a synergy of diverse disease exposures centrally regulating the miR-130/301-ECM remodeling axis may also be essential to the manifestation of both heritable and non-heritable forms of PAH alike.

Our findings also offer insight at the level of individual genes linked to heritable PAH, as their complete pathogenic actions have not been fully defined. This is particularly true for recently identified effectors ATP13A3, CBLN2, GCN2, SOX17, and TBX4 [2,5,7]. Our data not only link their functions to miR-130/301 biology and matrix remodeling but also define a cell-type specificity among PAAFs, PAECs, and PASMCs in many of those activities. As specific examples, ATP13A3 is a poorly characterized P-type ATPase. Although the precise substrate specificity is unknown, ATP13A3 plays a role in polyamine transport [38]. Previously, it was shown that loss of ATP13A3 inhibits proliferation of endothelial cells and increases apoptosis in serum-deprived conditions [5]. Consistent with the previously reported role of miR-130/301 family on context-dependent endothelial apoptosis [18], and with the paradigm that endothelial apoptosis is a major trigger for the initiation of PAH [39], we now have demonstrated that depletion of ATP13A3 induces miR-130/301 expression and forced miR-130a expression reciprocally decreases ATP13A3 (Figure 3B,E), suggesting a positive feedback loop consistent with promoting endothelial cell apoptosis and PAH. SOX17 encodes the SRY-box containing transcription factor 17 , which plays a fundamental role in angiogenesis and arteriovenous differentiation [40,41]. Moreover, conditional deletion of SOX17 in mesenchymal progenitors has been reported to impair formation of lung microvessels [42]. Adding to these insights, we observed that a SOX17-ECM remodeling-miR-130/301 axis is active in both PAAFs and PAECs but not in PASMCs (Figures 1 and 4). CBLN2 belongs to the cerebellin gene family, a group of secreted neuronal glycoproteins (Cbln1-4) [43]. CBLN2 has been previously reported to be expressed mainly in the brain but has also be found in PAH lung vasculature, particularly in diseased PAECs [9]. Consistent with these observations, we found that both matrix stiffening (Figure 1) and forced miR-130a expression induced CBLN2 expression (Figure 3). In turn, inhibition of CBLN2 activated the expression of the PPAR $\gamma$-APOE-LRP8 gene axis (Figure 4). Together, these results suggest that CBLN2 activation induces the miR-130/301-ECM remodeling feedback loop. Finally, TBX4 encodes the early embryonic transcription factor T-box gene 4. T-box genes are transcription factors known to be involved in several developmental and cardiovascular diseases [44]. Recently, TBX4 was found to play an important role in lung fibrosis in mice via its actions in $\alpha \mathrm{SMA}+$ myofibroblasts and COL1 $1 \alpha 1+$ fibroblasts [45]. Consistent with its role in fibrosis, we found that TBX4 knockdown decreased COL1A1, COL3A1, and LOX expression (Figure 4). While these results may seem contradictory with the fact that missense mutations (e.g., loss-of-function) in TBX are associated with PAH, it is important to note that TBX4 mutations are associated with childhood-onset $\mathrm{PAH}$, but the prevalence of PAH in adults with TBX4 mutation 
carriers is low [7]. Given the role of TBX4 during embryonic development and airway branching, it is tempting to speculate that while germline mutations of TBX4 increase the prevalence of PAH, somatic mutation of TBX4 could slow PAH progression by blunting ECM remodeling. Clearly, future work is necessary to delve more completely into these new mechanistic connections, and by limiting our analyses to known members of these previously identified pathways, we acknowledge a certain level of confirmation bias to this study. Nonetheless, our data offer a much-needed roadmap for delineating the miR-130/301-dependent overlap of these individual genes associated with heritable PAH.

While our findings indicate a new set of functional connections among the genes of heritable PAH with miR-130/301 and vascular stiffness, there are a number of concepts relevant to fundamental pathogenesis and applied therapeutic development that await exploration. First, our study utilized artificial means of forcing and inhibiting gene expression, and our readouts were simple quantification of gene transcript levels. Notably, the majority of genetic mutations reported to be associated with PAH are missense single nucleotide mutations, frameshift mutations (deletions or insertions), and nonsense mutations (stop codon gains), suggesting that these are associated with loss-of-function [2]. However, siRNA knockdown may not fully recapitulate the molecular underpinning of these genetic mutations, particularly if the relationship of these genes to miR-130/301 and matrix remodeling depend on precise gene "dosage." As such, future work will be necessary to study the functional impact of individual mutations and polymorphisms linked to PAH - potentially through use of inducible pluripotent stem cell technology and/or CRISPR/Cas9 technology [46] —and how the specific levels of altered expression that result may interact with the miR-130/301-ECM network. Second, our study only focused on pulmonary vascular cell types. There exists an emerging notion that several extrapulmonary organs (i.e., heart, skeletal muscle, gut, and adipose tissue, among others) demonstrate vascular and metabolic abnormalities in $\mathrm{PAH}$, suggesting a systemic, rather than exclusively pulmonary vascular, disease [47-50]. Failure of the right ventricle (RV) in PAH has been particularly linked to fibrosis. Furthermore, PPAR $\gamma$ activation has been shown to reverse these epigenetic, transcriptional, and metabolic alterations in the RV and prevent PAH development in rodent models of PAH [51]. As such, it remains an intriguing question whether factors associated with heritable PAH can also activate this miR-130/301-dependent positive feedback loop in the RV and whether targeted therapy that modulates this axis should also directly address RV failure. Finally, given the striking convergence of actions of multiple genes implicated in heritable PAH, there is a compelling possibility of synergistic therapeutic effects of a rational combination of pharmacologic PPAR $\gamma$ activation along with the unique application of shortmer technology to repress the miR-130/301 family. However, due to the varying levels of cell-type specificity and gene function overlap defined in our study, we also see an increasing need to predict and more precisely target such therapies to the target site(s) and molecular circuitry of interest. Consequently, we envision that further experimental validation of PAH network architecture, as led by computational prediction strategies (such as in Figure 5), may have a substantial impact on such systems pharmacology methods in PAH in the future.

In sum, we report that a network of upstream factors genetically linked to PAH converges upon the miR-130/301-PPAR $\gamma$-LRP8 axis with implications for controlling matrix remodeling and pulmonary vascular disease manifestation. While these results await validation in vivo, these functional relationships emphasize the causative importance of matrix and vascular stiffening in $\mathrm{PAH}$ and suggest a set of new and even surprising implications for how heritability and genetic penetrance in PAH may manifest over time.

\section{Materials and Methods}

\subsection{Cell Culture and Cell Culture Reagents}

Primary human pulmonary arterial endothelial cells (PAECs) were grown in EGM-2 cell culture media (Lonza, Basel, Switzerland), and experiments were performed at passages 3 to 6 . Primary human pulmonary arterial smooth muscle cells (PASMCs) were cultured in SmGM-2 cell culture media 
(Lonza), and experiments were performed at passages 3 to 9. Primary human pulmonary arterial adventitial fibroblast cells (PAAFs) were purchased (Sciencell Research Laboratories, Carlsbad, CA, USA) and grown in FGM cell culture media (Lonza). Experiments were performed at passages 3 to 6 . At baseline, cultured cells were grown in collagen-coated plastic $(50 \mu \mathrm{g} / \mathrm{mL})$ at $37^{\circ} \mathrm{C}$ in a humidified $5 \% \mathrm{CO}_{2}$ atmosphere. Collagen-coated hydrogel was purchased from Matrigen (Brea, CA, USA).

\subsection{Oligonucleotides and Transfection}

Pre-miRNA oligonucleotides (pre-miR-130a, negative control pre-miR-NC1, and premiR-NC2) and custom-designed tiny LNA oligonucleotides (tiny-130: $5^{\prime}$-ATTGCACT- $3^{\prime}$ and tiny-NC: 5'-TCATACTA-3') were purchased from Thermo Fisher Scientific (Waltham, MA, USA) and Exiqon (Qiagen, Germantown, MD, USA), respectively. PAAFs, PAECs, and PASMCs were plated in collagen-coated plastic $(50 \mu \mathrm{g} / \mathrm{mL})$ and transfected $24 \mathrm{~h}$ later at $70-80 \%$ confluence using pre-miRNA $(5 \mathrm{nM})$, tiny-LNA $(20 \mathrm{nM})$, or siRNA $(25 \mathrm{nM})$ and Lipofectamine 2000 reagent (Thermo Fisher Scientific), according to the manufacturers' instructions. Eight hours after transfection, cells were trypsinized and re-plated on hydrogel.

\subsection{Messenger RNA and miRNA Extraction}

Cells were homogenized in $1 \mathrm{ml}$ of QiaZol reagent (Qiagen, Germantown, MD, USA). Total RNA content, including small RNAs, was extracted using the miRNeasy kit (Qiagen) according to the manufacturer's instructions. Total RNA concentration was determined using a ND-1000 micro-spectrophotometer (NanoDrop Technologies, Wilmington, DE, USA).

\subsection{Quantitative RT-PCR of Mature miRNAs}

As we described previously [19], mature miRNA expression was evaluated using TaqMan MicroRNA Assays (Thermo Fisher Scientific) and the Applied Biosystems 7900HT Fast Real Time PCR device (Thermo Fisher Scientific). Expression levels were normalized to RNU48 and calculated using the comparative $\mathrm{Ct}$ method $\left(2^{-\Delta \Delta \mathrm{Ct}}\right)$. In order to ensure biological relevance, only $\mathrm{Ct}$ values $<35$ were utilized in the analysis.

\subsection{Quantitative RT-PCR of Messenger RNAs}

Messenger RNAs were reverse transcribed using the Multiscript RT kit (Thermo Fisher Scientific) to generate cDNA. cDNA was amplified via fluorescently labeled Taqman primer sets using an Applied Biosystems 7900HT Fast Real Time PCR device (Thermo Fisher Scientific). Fold-change of RNA species was calculated using the formula $\left(2^{-\Delta \Delta C t}\right)$, normalized to RPLP0 expression. In order to ensure biological relevance, only $\mathrm{Ct}$ values $<35$ were utilized in the analysis.

\subsection{Computational Gene Network Analysis}

A network analysis was devised to visualize functional interactions among factors genetically associated with PAH and known miR-130/301 target genes, PH genes, and fibrosis genes. First, a consolidated interactome $(\mathrm{CI})$ was constructed using gene and protein interaction data from public databases, including DIP, BioGRID, Corum, InnateDB, IntAct, MINT, and MatrixDB [24-30]. Second, we identified $\mathrm{PH}$-relevant genes in the $\mathrm{CI}$ by finding genes (nodes) common to a defined $\mathrm{PH}$ gene network. To define this PH Network, we curated a list of genes known to play a role in pulmonary hypertension from a literature search [18] and from the Pulmonary Arterial Hypertension Knowledge Base (PAHKB) [23]. These seed genes were mapped onto the CI, and the largest connected component (LCC) was identified. Additional first-degree interactors from the interactome were iteratively incorporated into the LCC that would include otherwise disconnected PH seed genes. This expansion led to the PH network and consisted of 3139 interactions among 833 genes, 370 of which were common to the literature search and PAHKB. Third, to identify fibrosis-relevant genes, we utilized a previously 
constructed Fibrosis Network (1459 interactions among 350 genes) [22], with updated modifications via inclusion of YAP/TAZ-dependent fibrotic factors in PAH, as we reported [10]. With these available networks (the CI, PH Network, and Fibrosis Network), functional interactions of the factors associated with heritable PAH and target genes of miR-130/301 were then mapped into the $\mathrm{PH}$ and Fibrosis networks. Specifically, we sought to identify the largest connected component (LCC) connecting genes associated with heritable PAH and miR-130/301 gene targets. Additional first-degree interactors from the CI were iteratively incorporated into this LCC that would include otherwise disconnected genes of heritable PAH and miR-130/301 target genes. The final LCC included 109 genes and 694 interactions.

Two rounds of gene set enrichment analysis (GSEA) [31] were performed on this LCC. The first analysis included all 109 genes in the expanded network. The second included only the 45 genes not common to either the PH Network or Fibrosis Network. Annotations were acquired from The Gene Ontology [52,53], KEGG [54], REACTOME [55,56], and BioCarta [57].

\subsection{Statistical Analysis}

Cell culture experiments were performed at least three times. Each independent experiment was performed in triplicate, results of which were averaged for further statistical analysis. Paired samples were compared using a 2-tailed Student's $t$-test for normally distributed data, while Mann-Whitney U non-parametric testing was used for non-normally distributed data. For comparisons among groups, one-way ANOVA and post-hoc Tukey testing was performed. A P-value less than 0.05 was considered significant. No samples were excluded.

Supplementary Materials: Supplementary materials can be found at http:/ /www.mdpi.com/1422-0067/19/8/ 2289/s1.

Author Contributions: T.B. and S.Y.C. conceived and designed the experiments. T.B. performed the experiments. A.L.H. performed the computational analyses. T.B. and S.Y.C. wrote the manuscript. All authors participated in interpreting the results and revising the manuscript.

Funding: This work was supported by NIH grants R01 HL124021, HL 122596, HL 138437, and UH2 TR002073 as well as the AHA grant 18EIA33900027 (S.Y.C.).

Acknowledgments: S.Y.C. has served as a consultant for Actelion (Significant), Gilead, Pfizer, Aerpio, and Vivus (Modest). Patent applications (S.Y.C. and T.B.) have been filed regarding microRNA and metabolic therapeutics in pulmonary hypertension.

\section{References}

1. Farber, H.; Loscalzo, J. Pulmonary arterial hypertension. N. Engl. J. Med. 2004, 351, 1655-1665. [CrossRef] [PubMed]

2. Austin, E.D.; Loyd, J.E. The genetics of pulmonary arterial hypertension. Circ. Res. 2014, 115, $189-202$. [CrossRef] [PubMed]

3. Simonneau, G.; Gatzoulis, M.A.; Adatia, I.; Celermajer, D.; Denton, C.; Ghofrani, A.; Gomez Sanchez, M.A.; Krishna Kumar, R.; Landzberg, M.; Machado, R.F.; et al. Updated clinical classification of pulmonary hypertension. J. Am. Coll. Cardiol. 2013, 62, D34-D41. [CrossRef] [PubMed]

4. Girerd, B.; Weatherald, J.; Montani, D.; Humbert, M. Heritable pulmonary hypertension: From bench to bedside. Eur. Respir. Rev. 2017, 26, 170037. [CrossRef] [PubMed]

5. Graf, S.; Haimel, M.; Bleda, M.; Hadinnapola, C.; Southgate, L.; Li, W.; Hodgson, J.; Liu, B.; Salmon, R.M.; Southwood, M.; et al. Identification of rare sequence variation underlying heritable pulmonary arterial hypertension. Nat. Commun. 2018, 9, 1416. [CrossRef] [PubMed]

6. Chung, W.K.; Sampson, K.S.; Kass, R.S. A novel channelopathy in pulmonary arterial hypertension. N. Engl. J. Med. 2013, 369, 2162. [CrossRef] [PubMed]

7. Kerstjens-Frederikse, W.S.; Bongers, E.M.; Roofthooft, M.T.; Leter, E.M.; Douwes, J.M.; Van Dijk, A.; Vonk-Noordegraaf, A.; Dijk-Bos, K.K.; Hoefsloot, L.H.; Hoendermis, E.S.; et al. TBX4 mutations (small patella syndrome) are associated with childhood-onset pulmonary arterial hypertension. J. Med. Genet. 2013, 50, 500-506. [CrossRef] [PubMed] 
8. $\quad$ Eyries, M.; Montani, D.; Girerd, B.; Perret, C.; Leroy, A.; Lonjou, C.; Chelghoum, N.; Coulet, F.; Bonnet, D.; Dorfmuller, P.; et al. EIF2AK4 mutations cause pulmonary veno-occlusive disease, a recessive form of pulmonary hypertension. Nat. Genet. 2014, 46, 65. [CrossRef] [PubMed]

9. Germain, M.; Eyries, M.; Montani, D.; Poirier, O.; Girerd, B.; Dorfmuller, P.; Coulet, F.; Nadaud, S.; Maugenre, S.; Guignabert, C.; et al. Genome-wide association analysis identifies a susceptibility locus for pulmonary arterial hypertension. Nat. Genet. 2013, 45, 518-521. [CrossRef] [PubMed]

10. Bertero, T.; Cotrill, K.A.; Lu, Y.; Haeger, C.M.; Dieffenbach, P.; Annis, S.; Hale, A.; Bhat, B.; Kaimal, V.; Zhang, Y.Y.; et al. Matrix remodeling promotes pulmonary hypertension through feedback mechanoactivation of the YAP/TAZ-miR-130/301 circuit. Cell Rep. 2015, 13, 1016-1032. [CrossRef] [PubMed]

11. Liu, F.; Haeger, C.M.; Dieffenbach, P.B.; Sicard, D.; Chrobak, I.; Coronata, A.M.; Suarez Velandia, M.M.; Vitali, S.; Colas, R.A.; Norris, P.C.; et al. Distal vessel stiffening is an early and pivotal mechanobiological regulator of vascular remodeling and pulmonary hypertension. JCI Insight 2016, 1, 8. [CrossRef] [PubMed]

12. Mahapatra, S.; Nishimura, R.A.; Sorajja, P.; Cha, S.; McGoon, M.D. Relationship of pulmonary arterial capacitance and mortality in idiopathic pulmonary arterial hypertension. J. Am. Coll. Cardiol. 2006, 47, 799-803. [CrossRef] [PubMed]

13. Gan, C.T.; Lankhaar, J.W.; Westerhof, N.; Marcus, J.T.; Becker, A.; Twisk, J.W.; Boonstra, A.; Postmus, P.E.; Vonk-Noordegraaf, A. Noninvasively assessed pulmonary artery stiffness predicts mortality in pulmonary arterial hypertension. Chest 2007, 132, 1906-1912. [CrossRef] [PubMed]

14. Hunter, K.S.; Lee, P.F.; Lanning, C.J.; Ivy, D.D.; Kirby, K.S.; Claussen, L.R.; Chan, K.C.; Shandas, R. Pulmonary vascular input impedance is a combined measure of pulmonary vascular resistance and stiffness and predicts clinical outcomes better than pulmonary vascular resistance alone in pediatric patients with pulmonary hypertension. Am. Heart J. 2008, 155, 166-174. [CrossRef] [PubMed]

15. Tabima, D.M.; Roldan-Alzate, A.; Wang, Z.; Hacker, T.A.; Molthen, R.C.; Chesler, N.C. Persistent vascular collagen accumulation alters hemodynamic recovery from chronic hypoxia. J. Biomech. 2012, 45, 799-804. [CrossRef] [PubMed]

16. Lau, E.M.; Iyer, N.; Ilsar, R.; Bailey, B.P.; Adams, M.R.; Celermajer, D.S. Abnormal pulmonary artery stiffness in pulmonary arterial hypertension: In vivo study with intravascular ultrasound. PLoS ONE 2012, 7, e33331. [CrossRef] [PubMed]

17. Bertero, T.; Cottrill, K.; Krauszman, A.; Lu, Y.; Annis, S.; Hale, A.; Bhat, B.; Waxman, A.B.; Chau, B.N.; Kuebler, W.M.; et al. The microRNA-130/301 family controls vasoconstriction in pulmonary hypertension. J. Biol. Chem. 2014, 290, 2069-2085. [CrossRef] [PubMed]

18. Bertero, T.; Lu, Y.; Annis, S.; Hale, A.; Bhat, B.; Saggar, R.; Saggar, R.; Wallace, W.D.; Ross, D.J.; Vargas, S.O.; et al. Systems-level regulation of microRNA networks by miR-130/301 promotes pulmonary hypertension. J. Clin. Invest. 2014, 124, 3514-3528. [CrossRef] [PubMed]

19. Bertero, T.; Oldham, W.M.; Cottrill, K.A.; Pisano, S.; Vanderpool, R.R.; Yu, Q.; Zhao, J.; Tai, Y.; Tang, Y.; Zhang, Y.Y.; et al. Vascular stiffness mechanoactivates YAP/TAZ-dependent glutaminolysis to drive pulmonary hypertension. J. Clin. Invest. 2016, 126, 3313-3335. [CrossRef] [PubMed]

20. Negi, V.; Chan, S.Y. Discerning functional hierarchies of microRNAs in pulmonary hypertension. JCI Insight 2017, 2, e91327. [CrossRef] [PubMed]

21. Friedman, R.C.; Farh, K.K.; Burge, C.B.; Bartel, D.P. Most mammalian mRNAs are conserved targets of microRNAs. Genome Res. 2009, 19, 92-105. [CrossRef] [PubMed]

22. Bertero, T.; Cottrill, K.A.; Annis, S.; Bhat, B.; Gochuico, B.R.; Osorio, J.C.; Rosas, I.; Haley, K.J.; Corey, K.E.; Chung, R.T.; et al. A YAP/TAZ-miR-130/301 molecular circuit exerts systems-level control of fibrosis in a network of human diseases and physiologic conditions. Sci. Rep. 2015, 5, 18277. [CrossRef] [PubMed]

23. Zhao, M.; Austin, E.D.; Hemnes, A.R.; Loyd, J.E.; Zhao, Z. An evidence-based knowledgebase of pulmonary arterial hypertension to identify genes and pathways relevant to pathogenesis. Mol. Biosyst. 2014, 10, 732-740. [CrossRef] [PubMed]

24. Salwinski, L.; Miller, C.S.; Smith, A.J.; Pettit, F.K.; Bowie, J.U.; Eisenberg, D. The Database of Interacting Proteins: 2004 update. Nucleic Acids Res. 2004, 32, D449-D451. [CrossRef] [PubMed]

25. Chatr-Aryamontri, A.; Breitkreutz, B.J.; Oughtred, R.; Boucher, L.; Heinicke, S.; Chen, D.; Stark, C.; Breitkreutz, A.; Kolas, N.; O'Donnell, L.; et al. The BioGRID interaction database: 2015 update. Nucleic Acids Res. 2015, 43, D470-D478. [CrossRef] [PubMed] 
26. Ruepp, A.; Waegele, B.; Lechner, M.; Brauner, B.; Dunger-Kaltenbach, I.; Fobo, G.; Frishman, G.; Montrone, C.; Mewes, H.W. CORUM: The comprehensive resource of mammalian protein complexes 2009. Nucleic Acids Res. 2010, 38, D497-D501. [CrossRef] [PubMed]

27. Breuer, K.; Foroushani, A.K.; Laird, M.R.; Chen, C.; Sribnaia, A.; Lo, R.; Winsor, G.L.; Hancock, R.E.; Brinkman, F.S.; Lynn, D.J. InnateDB: Systems biology of innate immunity and beyond-Recent updates and continuing curation. Nucleic Acids Res. 2013, 41, D1228-D1233. [CrossRef] [PubMed]

28. Orchard, S.; Ammari, M.; Aranda, B.; Breuza, L.; Briganti, L.; Broackes-Carter, F.; Campbell, N.H.; Chavali, G.; Chen, C.; del-Toro, N.; et al. The MIntAct project-IntAct as a common curation platform for 11 molecular interaction databases. Nucleic Acids Res. 2014, 42, D358-D363. [CrossRef] [PubMed]

29. Licata, L.; Briganti, L.; Peluso, D.; Perfetto, L.; Iannuccelli, M.; Galeota, E.; Sacco, F.; Palma, A.; Nardozza, A.P.; Santonico, E.; et al. MINT, the molecular interaction database: 2012 update. Nucleic Acids Res. 2012, 40, D857-D861. [CrossRef] [PubMed]

30. Launay, G.; Salza, R.; Multedo, D.; Thierry-Mieg, N.; Ricard-Blum, S. MatrixDB, the extracellular matrix interaction database: Updated content, a new navigator and expanded functionalities. Nucleic Acids Res. 2015, 43, D321-D327. [CrossRef] [PubMed]

31. Subramanian, A.; Tamayo, P.; Mootha, V.; Mukherjee, S.; Ebert, B.; Gillette, M.; Paulovich, A.; Pomeroy, S.; Golub, T.; Lander, E.; et al. Gene set enrichment analysis: A knowledge-based approach for interpreting genome-wide expression profiles. Proc. Natl. Acad. Sci. USA 2005, 102, 15545-15550. [CrossRef] [PubMed]

32. Meloche, J.; Pflieger, A.; Vaillancourt, M.; Paulin, R.; Potus, F.; Zervopoulos, S.; Graydon, C.; Courboulin, A.; Breuils-Bonnet, S.; Tremblay, E.; et al. Role for DNA damage signaling in pulmonary arterial hypertension. Circulation 2014, 129, 786-797. [CrossRef] [PubMed]

33. Ranchoux, B.; Meloche, J.; Paulin, R.; Boucherat, O.; Provencher, S.; Bonnet, S. DNA Damage and Pulmonary Hypertension. Int. J. Mol. Sci. 2016, 17, 990. [CrossRef] [PubMed]

34. Dupont, S.; Morsut, L.; Aragona, M.; Enzo, E.; Giulitti, S.; Cordenonsi, M.; Zanconato, F.; Le Digabel, J.; Forcato, M.; Bicciato, S.; et al. Role of YAP/TAZ in mechanotransduction. Nature 2011, 474, 179-183. [CrossRef] [PubMed]

35. Chan, S.Y.; Loscalzo, J. Pathogenic mechanisms of pulmonary arterial hypertension. J. Mol. Cell. Cardiol. 2008, 44, 14-30. [CrossRef] [PubMed]

36. Pousada, G.; Baloira, A.; Valverde, D. Complex inheritance in Pulmonary Arterial Hypertension patients with several mutations. Sci. Rep. 2016, 6, 33570. [CrossRef] [PubMed]

37. Hadinnapola, C.; Bleda, M.; Haimel, M.; Screaton, N.; Swift, A.J.; Dorfmuller, P.; Preston, S.D.; Southwood, M.; Hernandez-Sanchez, J.; Martin, J.; et al. Phenotypic Characterisation of EIF2AK4 Mutation Carriers in a Large Cohort of Patients Diagnosed Clinically with Pulmonary Arterial Hypertension. Circulation 2017, 136, 2022-2033. [CrossRef] [PubMed]

38. Madan, M.; Patel, A.; Skruber, K.; Geerts, D.; Altomare, D.A.; Iv, O.P. ATP13A3 and caveolin-1 as potential biomarkers for difluoromethylornithine-based therapies in pancreatic cancers. Am. J. Cancer Res. 2016, 6, 1231-1252. [PubMed]

39. Michelakis, E.D. Spatio-temporal diversity of apoptosis within the vascular wall in pulmonary arterial hypertension: Heterogeneous BMP signaling may have therapeutic implications. Circ. Res. 2006, 98, 172-175. [CrossRef] [PubMed]

40. Matsui, T.; Kanai-Azuma, M.; Hara, K.; Matoba, S.; Hiramatsu, R.; Kawakami, H.; Kurohmaru, M.; Koopman, P.; Kanai, Y. Redundant roles of Sox17 and Sox18 in postnatal angiogenesis in mice. J. Cell Sci. 2006, 119, 3513-3526. [CrossRef] [PubMed]

41. Corada, M.; Orsenigo, F.; Morini, M.F.; Pitulescu, M.E.; Bhat, G.; Nyqvist, D.; Breviario, F.; Conti, V.; Briot, A.; Iruela-Arispe, M.L.; et al. Sox17 is indispensable for acquisition and maintenance of arterial identity. Nat. Commun. 2013, 4, 2609. [CrossRef] [PubMed]

42. Lange, A.W.; Haitchi, H.M.; LeCras, T.D.; Sridharan, A.; Xu, Y.; Wert, S.E.; James, J.; Udell, N.; Thurner, P.J.; Whitsett, J.A. Sox17 is required for normal pulmonary vascular morphogenesis. Dev. Biol. 2014, 387, 109-120. [CrossRef] [PubMed]

43. Yiangou, Y.; Burnet, P.; Nikou, G.; Chrysanthou, B.J.; Bloom, S.R. Purification and characterisation of cerebellins from human and porcine cerebellum. J. Neurochem. 1989, 53, 886-889. [CrossRef] [PubMed]

44. Arora, R.; Metzger, R.J.; Papaioannou, V.E. Multiple roles and interactions of Tbx4 and Tbx5 in development of the respiratory system. PLoS Genet. 2012, 8, e1002866. [CrossRef] [PubMed] 
45. Xie, T.; Liang, J.; Liu, N.; Huan, C.; Zhang, Y.; Liu, W.; Kumar, M.; Xiao, R.; D'Armiento, J.; Metzger, D.; et al. Transcription factor TBX4 regulates myofibroblast accumulation and lung fibrosis. J. Clin. Invest. 2016, 126, 3063-3079. [CrossRef] [PubMed]

46. Gu, M.; Shao, N.Y.; Sa, S.; Li, D.; Termglinchan, V.; Ameen, M.; Karakikes, I.; Sosa, G.; Grubert, F.; Lee, J.; et al. Patient-Specific iPSC-Derived Endothelial Cells Uncover Pathways that Protect against Pulmonary Hypertension in BMPR2 Mutation Carriers. Cell Stem Cell 2017, 20, 490-504. [CrossRef] [PubMed]

47. Sutendra, G.; Michelakis, E.D. The metabolic basis of pulmonary arterial hypertension. Cell Metab. 2014, 19, 558-573. [CrossRef] [PubMed]

48. Zamanian, R.T.; Hansmann, G.; Snook, S.; Lilienfeld, D.; Rappaport, K.M.; Reaven, G.M.; Rabinovitch, M.; Doyle, R.L. Insulin resistance in pulmonary arterial hypertension. Eur. Respir. J. 2009, 33, 318-324. [CrossRef] [PubMed]

49. Hansmann, G.; Rabinovitch, M. The protective role of adiponectin in pulmonary vascular disease. Am. J. Physiol. Lung Cell. Mol. Physiol. 2010, 298, L1-L2. [CrossRef] [PubMed]

50. Ranchoux, B.; Bigorgne, A.; Hautefort, A.; Girerd, B.; Sitbon, O.; Montani, D.; Humbert, M.; Tcherakian, C.; Perros, F. Gut-Lung Connection in Pulmonary Arterial Hypertension. Am. J. Respir. Cell Mol. Biol. 2017, 56, 402-405. [CrossRef] [PubMed]

51. Legchenko, E.; Chouvarine, P.; Borchert, P.; Fernandez-Gonzalez, A.; Snay, E.; Meier, M.; Maegel, L.; Mitsialis, S.A.; Rog-Zielinska, E.A.; Kourembanas, S.; et al. PPAR $\gamma$ agonist pioglitazone reverses pulmonary hypertension and prevents right heart failure via fatty acid oxidation. Sci. Transl. Med. 2018, 10, eaao0303. [CrossRef] [PubMed]

52. The Gene Ontology Consortium. Expansion of the Gene Ontology knowledgebase and resources. Nucleic Acids Res. 2017, 45, D331-D338. [CrossRef]

53. Ashburner, M.; Ball, C.A.; Blake, J.A.; Botstein, D.; Butler, H.; Cherry, J.M.; Davis, A.P.; Dolinski, K.; Dwight, S.S.; Eppig, J.T.; et al. Gene ontology: Tool for the unification of biology. The Gene Ontology Consortium. Nat. Genet. 2000, 25, 25-29. [CrossRef] [PubMed]

54. Kanehisa, M.; Furumichi, M.; Tanabe, M.; Sato, Y.; Morishima, K. KEGG: New perspectives on genomes, pathways, diseases and drugs. Nucleic Acids Res. 2017, 45, D353-D361. [CrossRef] [PubMed]

55. Fabregat, A.; Sidiropoulos, K.; Garapati, P.; Gillespie, M.; Hausmann, K.; Haw, R.; Jassal, B.; Jupe, S.; Korninger, F.; McKay, S.; et al. The Reactome pathway Knowledgebase. Nucleic Acids. Res. 2016, 44, D481-D487. [CrossRef] [PubMed]

56. Croft, D.; Mundo, A.F.; Haw, R.; Milacic, M.; Weiser, J.; Wu, G.; Caudy, M.; Garapati, P.; Gillespie, M.; Kamdar, M.R.; et al. The Reactome pathway knowledgebase. Nucleic Acids Res. 2014, 42, D472-D477. [CrossRef] [PubMed]

57. Nishimura, D. BioCarta. Bio. Softw. Int. Rep. 2001, 2, 117-120. [CrossRef] 